\title{
Visual perception of five-year-old English-speaking children in Bloemfontein using the Beery VMI-6, DTVP-3 and TVPS-3
}

\author{
Marieta Visser, MSc (OT) (Wits) \\ Department of Occupational Therapy, Faculty of Health Sciences, University of the Free State, Bloemfontein, South Africa
}

Riette Nel, M MedSc (Biostatistics) (UFS)

Department of Biostatistics, Faculty of Health Sciences, University of the Free State, Bloemfontein, South Africa

\section{Tasmia Jansen, B OT (UFS)* \\ Lauren Kinmont, B OT (UFS)* \\ Susanna Terblanché, B OT (UFS)* \\ Jorika van Wyk, B OT (UFS)*}

* $4^{\text {th }}$-year students, Department of Occupational Therapy, Faculty of Health Sciences, University of the Free State, Bloemfontein, South Africa at the time of study.

Background and aim: The Beery-Buktenica Developmental Test of Visual-motor Integration $6^{\text {th }}$ edition (Beery VMI-6), Development Test of Visual Perception $3^{\text {rd }}$ edition (DTVP-3) and Test of Visual Perceptual Skills $3^{\text {rd }}$ edition (TVPS-3) are the latest editions of three commonly used visual perception tests, not yet widely studied in South Africa. Research on previous editions of these tests on South African children has been reported. The aim of this study was to investigate the new versions of the tests that have more recently become available in South Africa.

Methods: This article reports on a quantitative, cross-sectional investigation into the visual perception and visual-motor performance of five-year-old English-speaking children, by using the three tests mentioned above. Sixty-eight (68) children were selected from English Language of Learning and Teaching (ELOLT) Bloemfontein schools. Children completed the tests according to the prescribed procedures, in a specific order and with adequate breaks between tests.

Results: The findings showed that the children's performance on the Beery VMI-6 and DTVP-3 compared well to the American normative sample. However, all the children in this study scored below average in all the subtests, with the exception of the test of visual memory, and all of the composite TVPS-3 scores.

Conclusions: The TVPS-3 should be used with caution and preferably not as the only visual perception measuring instrument. Occupational therapists in South Africa should consider the suitability of the tests and develop instruments specifically appropriate for the South African context.

Key words: visual perceptual tests; visual-motor performance; five-year-old children; Beery VMI-6; DTVP-3; TVPS-3

\section{INTRODUCTION}

Visual perceptual and visual-motor abilities play an important role in the performance of daily children's activities such as building a puzzles, dressing, reading and writing. It is important for occupational therapists to identify with which of these aspects children may have difficulties before therapeutic intervention is implemented. Standardised measuring instruments provide occupational therapists and other professionals with a reliable measure of a child's perceptual abilities, to guide goal setting and evaluate intervention outcomes'.

Internationally, previously released versions of the Beery-Buktenica Developmental Test of Visual-motor Integration (Beery-VMI), the Developmental Test of Visual Perception (DTVP) and the Test of Visual Perceptual Skills (TVPS) are among the most commonly used measuring instruments used by occupational therapists to assess the visual perception and visual-motor abilities of children ${ }^{2-4}$. Even among South African occupational therapists, these three tests are mostly preferred, despite all of them having been developed and standardised on an American sample $e^{5}$ The latest editions of these three instruments have been released and are now available for use in South Africa. The Beery VMI-6 was published in $2010^{6}$, the DTVP-3 in $2014^{7}$ and the TVPS-3 in 2006!.

Over an extended period, research on the previous versions of these three tests had been conducted in numerous countries across the world ${ }^{2,3,9-24}$. Some of these studies also investigated aspects of the validity and reliability of the tests ${ }^{9-11}$. Cultural variations ${ }^{18}$ and the relationship between test results and aspects such as vision ${ }^{18,21}$ and achievement tests ${ }^{8}$, have been reported. Furthermore, a variety of age groups ${ }^{12}$ and specific populations, such as individuals with particular diagnoses and/or impairments, on whom these tests had been performed, have been described ${ }^{23-28}$. Comparative studies on subtests (such as VMI) of these tests have also been conducted ${ }^{29-30}$. A review of the available literature on the Beery VMI- 6 was published in $2012^{31}$. Despite the extensive research and published reports on earlier versions of these tests, limited research findings on the latest versions are currently available. 
With regard to the South African context, research on the previous versions of these tests have found unique differences in the South African population ${ }^{32-40}$. Differences in the performance of South African children as compared to the American norms were identified in the Beery VMI and DTVP-2, TVPS-R where children would score above, average or below average on certain subtests. These studies have been reported and applied to provide guidance to therapists in their clinical practice, to ensure that these instruments are used with care.

Only one study that investigated the latest versions of these tests used on a small sample of 48 South African children, was presented at the $35^{\text {th }}$ OTASA conference in $2016^{41}$. It is therefore imperative to question the suitability of administering these tests to describe the unique differences found among South African children, and to propose recommendations for practice when therapists convert to the latest editions and use these visual perception tests. The aim of this study was therefore to investigate the visual perceptual and visual-motor performance of five-year-old children attending English Language of Learning and Teaching (ELOLT) schools in Bloemfontein, using the Beery VMI-6, DTVP-3 and TVPS-3.

\section{Literature review}

Visual perception is the ability to identify, organise, attach meaning to and provide sense to what is seen'. It is a highly sophisticated and integrative ability that incorporates inter-related sub-skills such as visual-motor integration, and motor-reduced visual perception'. According to the Occupational Therapy Practice Framework ${ }^{42}$, visual perception is an occupational performance skill needed to support engagement and participation in daily life occupations and both visual perception and visual-motor integration are considered functionally important to childhood occupations. Visual perceptual skills can be assessed during clinical observations, informal assessment and by means of standardised measuring instruments ${ }^{43}$.

The lack of contextual-specific standardised assessment instruments is a universal problem in all fields of occupational therapy. In the field of paediatric practice, no comprehensive visual perceptual skills measuring instruments that have been standardised on a representative South African population, are currently available. The Early Childhood Developmental Criteria (ECDC) is used to assess visual perception and fine and gross motor aspects, and has been standardised for application in South Africa. However, the ECDC is only used on children between three and six years of $\mathrm{age}^{44}$. Consequently, occupational therapists use measuring instruments standardised in other countries to assess children's visual perceptual skills $s^{5,33,38}$.

Clinicians should be cautious about using an assessment instrument on individuals in communities, with cultures and languages different from the ones on which the instrument had been standardised ${ }^{18}$. Certain factors need to be taken into consideration with regard to this particular matter, and include the following:

* Research has shown that children from different cultures develop at different rates. Therefore, applying a set of norms from one culture to another could lead to the misrepresentation of the child's true developmental status ${ }^{45}$;

* Several studies have evaluated the performance of children from different countries and/or cultural groups using American paediatric standardised tests and identified differences in the outcomes $^{18}$;

* The level of difficulty of test items might vary depending on the child's prior experiences and exposure to educational settings ${ }^{20}$;

- Results obtained by means of inaccurate evaluations can lead to misinterpretations and implications on the intervention plan ${ }^{18}$;

* It can be an ethical issue if cultural bias is not considered when using these standardised tests ${ }^{46}$.

\section{Beery VMI}

The Beery is widely considered to be the best researched and most valid test of its kind ${ }^{6}$. The first edition of the Beery VMI test was published in 1967 and has been standardised six times up to
2010 , with a total of more than 13000 children being included in its development ${ }^{6}$. Hence it is considered as one of the most popular, economical and valid measuring instruments used by a wide variety of professionals ${ }^{8}$. The Beery can be administered to individuals and groups $2-100$ years of age in approximately $10-15$ minutes $^{6}$. It consists of three sections, namely the visual-motor integration subtest and the visual perception and motor coordination supplementary tests. The sixth edition of the Beery includes a new section on reports, research and other advances such as a section on VMI teaching tools ${ }^{6}$.

"For the reliability of a test as the Beery VMI requires that there will be adequate consistency in (I) the content of the items, (2) individuals' performance on the tests when it is re-administered, and (3) scoring performance by different examiners"6:103. The reliability of the Beery sixth edition is considered to exceed standards for the three above-mentioned aspects (for a test to be reliable it must approximate or exceed .80 ). With regard to the validity of the Beery VMI and its supplementary tests, content, concurrent, construct and predictive validity were examined and reported to measure at very high levels of between .80 and $.95^{6}$.

The Beery VMI has been claimed to be culture-free ${ }^{6}$. Brown and Hockey" "found that the Beery VMI-6 was suitable for use on the Australian population, as their findings did not show any cultural bias in an Australian context. However, Lim et al. ${ }^{18}$ regarded it as sensitive to culture, since several studies have shown differences in children's performance when compared to the American norms. For example, in a study conducted in Singapore using the fifth edition of the Beery VMI, a significant difference between the performance of the Singaporean pre-schoolers and the standardised sample of American children was found ${ }^{18}$. Unique differences were also observed among South African children ${ }^{32-35,39}$, such as: some of the SA population presenting with lower test performance ${ }^{35}$, or higher performance in the Motor Coordination subtest than the American normative sample ${ }^{33}$, and performance differences observed in environmentally disadvantaged ${ }^{34}$ and socio-economic status groups ${ }^{32}$.

\section{DTVP-3}

The first edition of the DTVP was published in I96I, revised into the DTVP-2 in 1993 and the third edition (DTVP-3) was improved and published in 20I4. The normative sample consisted of I, 035 children from 27 states in the USA. The DTVP-3 was designed for use with children 4 through 12 . The testing time will range from 20 to 40 minutes depending on the child's age and abilities. Reliability with regard to content, time and scorer, proved to exceed a coefficient of 0.80 . Three types of validity were established in the DTVP-3, namely content description, criterion-prediction and construct-identification ${ }^{7}$.

The DTVP-3 consists of five subtests. Some of the improvements in the third edition include the following ${ }^{7}$ :

* All-new normative data were collected in 2010 and 2011, recruiting a representative American sample (based on the 2010 USA Census Bureau).

* Three subtests were dropped, because the authors recognised shortcomings such as position in space, spatial relations and visual-motor speed. Position in space (PS) and Spatial Relations (SR) were dropped because the authors recognised that there were too few difficult items for children aged eight years and older. The Visual Motor speed (VMS) sub-tests was found to correlate poorly with other visual-motor integration subtest and therefore dropped.

* The age range has been extended to include 12-year-old children.

* The DTVP-3 consists of eye-hand coordination (EH), copying $(\mathrm{CO})$, figure-ground ( $\mathrm{FG})$, visual closure (VC) and form constancy $(F C)$ subtests.

Limited research has been done on the DTVP-3 after it was published in 2014. Brown and Murdolo ${ }^{15}$ stated the following in a review of the test: "The DTVP-3 is a reliable and valid instrument, 
although more research is needed to assess its psychometric properties and to validate its use in other cross-cultural contexts. It is a much improved version compared to its predecessor" $15: 35$. The construct validity of the DTVP-3 for the two constructs visualmotor integration and reduced visual perception were confirmed for a small (N-9I) sample of typical developing Australian children. However the copying subtest exhibited some factor complexity and further research is recommended ${ }^{47}$.

Studies done on the use of the previous edition (DTVP-2) in countries such as Hong Kong, showed that certain subtests were too easy for the children ${ }^{20}$. A factor found to have an influence on the lower difficulty level of these tests, was that Hong Kong children start to read and write at the age of five years ${ }^{20}$, which is a year or even two years earlier than most American children. Since South African children have to attend Grade I only in the year in which they turn seven ${ }^{48}$ they are also not always exposed to reading and writing activities from an early age. Similar results were found for children from Thailand, where children enter school at the age of three and, therefore, have early exposure to activities that encourage writing ${ }^{24}$.

Three South African studies reported that children's scores were significantly lower in the visual closure subtest and concluded that it was not a valid measuring tool to assess their visual closure abilities $^{38}$. In other subtests (copying, figure-ground and spatial relations), children scored above the American norm. The researchers emphasised the importance for occupational therapists to keep this information in mind "in order to interpret DTVP-2 results with care for the South African population" $38: 25$.

Therefore, it is clear that certain cultural experiences can affect the suitability of the use of the DTVP-2 in countries other than America. The researchers from Hong Kong concluded by stating that "...there is a need to ensure that norms for all tests are appropriate for the specific culture groups being assessed"20:41.

\section{TVPS-3}

The previous TVPS editions were published in 1982 (TVPS) ${ }^{49}$ and 1996 (TVPS-R) $)^{50}$, followed by a few other variations, with the TVPS3 being published in 2006'. The TVPS-3 provides new American stratified norms based on a sample of over 2000 children. It assesses an individual's visual perceptual abilities, requiring as little as possible motor involvement when making a response'. The TVPS-3 can be completed in approximately 30 minutes, and consists of seven subtests: visual discrimination (VD), visual memory (VM), spatial relations (SR), form constancy (FC), sequential memory (SM), figure-ground (FG) and visual closure (VC). Granting that the fourth edition, the TVPS- 4 was published in 2017 , it was not available to the researchers at the time of this study ${ }^{51}$.

The TVPS-3 has a high level of reliability because it provides a consistent measure, relatively free of error, the test content has a high level of homogeneity, provides consistent measurement from one testing to the next, and shows consistency by different examiners'. The three types of validity, namely content, criterion-related and construct were examined and proved to be appropriate to assess construct visual perception'.

A few structural changes that were made to the test include the following:

* The TVPS-3 is now one test, instead of two, for use with individuals $4-18$ years of age.

* The subtest structure changed to be more uniform - all subtests have a uniform length of 16 items each and two example items (instead of one), which can be used to teach the task.

* A new optional scoring component for the composite scores was included (basic processes, sequential processing and complex processing) to allow the examiner to evaluate related skills.

Only one study could be found on the use of the TVPS-3 in countries other than the USA. This study (aimed to investigate the convergent validity of the DTVP-2 with Beery VMI and TVPS-3) found that the TVPS-3 was suitable to use on the Australian popu- lation" ", showing that these visual perception tests that were standardised in America could generally be suitable for use in Australia.

\section{AIM}

The aim of this study was to describe the visual perception and visual-motor performance of five-year-old ( 5 years 6 months to 5 years II months) English-speaking children in Bloemfontein, South Africa, by using the Beery VMI-6, DTVP-3 and TVPS-3 measuring instruments' accepted norms. The objectives of the study were firstly, to describe and compare the Beery VMI-6, DTVP-3 and TVPS-3 visual-perceptual and visual motor subtests and secondly to investigate the gender differences in performance of the Beery VMI-6, DTVP-and TVPS-3 on a sample of five-year old Englishspeaking children in Bloemfontein.

\section{METHODOLOGY}

\section{Design}

A quantitative, cross-sectional study design was employed.

\section{Population and sampling}

Since the DTVP-2 was standardised in English and is not available in any other language, the study population focused on children who were able to understand and speak English. The study sample was therefore recruited from English Language of Learning and Teaching (ELOLT) public and private schools with Grade pre-R and Grade R classes in Bloemfontein. English is considered the language medium through which learning and teaching, including assessment occurs ${ }^{52}$ at these schools.

From the 22 schools identified through the Free State Department of Basic Education (personal communication; Corina Botha $16 / 02 / 2016$ ), seven schools were eligible to be included in the study. The remaining schools either were not suitable (e.g. did not have a grade $\mathrm{R}$ class), did not agree to participate, had insufficient contact details, or did not respond to phone calls and emails after several attempts to contact them. Written informed consent was obtained from the principals and the teachers involved at the eligible schools prior to the study.

The seven participating schools were contacted to obtain information with regard to the number of Grade pre-R and Grade R classes they had, how many children there were, how many were boys and girls, and how many of the children in these classes fell within the age bracket of this study.

Children were included in the study if they were aged between 5 years 6 months and 5 years II months of age, had been attending an ELOLT school since January 2016, and were able to speak and understand English. This specific age was selected as other similar studies had been done on the same age group, and therefore the findings of this study could be compared to those reported by those studies ${ }^{36,53}$.

Children were excluded from the study if any of the following aspects were indicated on the parent/caregiver questionnaire:

* the child had physical and/or cognitive disabilities or limitations due to a pathology that could negatively influence their participation during the tests;

* the child presented with any sensory, physical or emotional impairment or any condition that could influence their participation and/or test results;

* the child had been tested by means of the three tests within the preceding six months before the research was conducted;

* the child had received occupational therapy intervention or any other type of therapy, such as seeing a psychologist before the study;

* the parents/guardians did not give consent for the child's participation in the study; and

* the child did not assent to participate.

A total number of 187 potential children, of which 97 (5I.9\%) were boys, were identified. The parents/guardians of these 187 chil- 
dren received information letters and parent questionnaires. With only 122 of these questionnaires being completed and returned within the planned research execution time frame, the response rate was $65.2 \%$. However, 53 of these did not meet the inclusion/ exclusion criteria and one child was absent during data collection, giving a total of 68 children who participated in the study.

As a result of limited returned consent forms received from parents, limited time and finances available to resend questionnaires or send reminders to parents who had not returned the questionnaires, a convenience sampling method was used instead of the originally planned randomised sampling. Consequently, a minor amendment to the protocol was submitted and approved by the Health Sciences Research Ethics Committee of the University of the Free State.

\section{Data collection}

The fourth year occupational therapy student researchers, who collected the data, had theoretical and clinical training in the use of all three tests from their second year. To ensure that all the administration and scoring were done according to the prescribed guidelines, revised training and competency evaluation was done by the research supervisor (who is a qualified occupational therapist with paediatric experience in all three the tests), prior to data collection.

A pilot study was done on four children at two of the participating schools using the prescribed guidelines of each test. It was conducted in order to orientate each of the four student researchers and teachers to the research process, to determine how long the tests might take on average, to determine whether the children understood the assent forms, whether the study setting was appropriate, and to identify whether the break times between tests were sufficient. Since no adjustments were required to the prescribed administration and scoring of the tests, the data collected from the pilot study were included in the results of the main study.

Two student researchers were assigned per school and assessed four children individually per day. The allocated venue was prepared to ensure minimal distractions and was equipped with age-appropriate tables and chairs. After assent was obtained from the child, the TVPS- 3 was administered, after which the child received a 60 minute break during which he/she went back to class.

To enhance reliability, the three tests were administered in the same order, by all four researchers, in the same language (English) and according to the prescribed method of each instrument's Exam- iner's Manual 1,6,7. The researchers used the original tests - and scoring booklets to comply with copyright of the publishing companies.

The researchers used the corresponding tables in each instrument's Examiner's Manual ${ }^{1,6,7}$ to convert the raw scores of all the subtests to the standard scores for each of the three tests. Furthermore, to prevent possible clerical errors, the scoring of the three tests was verified on the test booklets by a researcher who did not conduct the test. After scores were verified, the raw- and standard scores were transferred to data score sheets by two researches and verified by the two other researches. Lastly, a biostatistician verified the data on the score sheets before data analysis.

\section{Data analysis}

Descriptive statistics, namely frequencies and percentages for categorical data and medians and percentiles for continuous data, were calculated per subtest/gender, and compared by means of $95 \%$ confidence intervals $(95 \% \mathrm{Cl})$ for the median differences.

The data management procedure that was used for this study is the filing and storing of the data within the Department of Occupational Therapy, in a locked cabinet where it will remain for fifteen years.

\section{Ethical considerations}

Approval for this study was obtained from the Health Science Research Ethics Committee of the Faculty of Health Sciences, University of the Free State (reference HSREC-S I5/20I6). To ensure ethical conduct, written permission was granted by the Free State Department of Basic Education, the participating schools' principals and class teachers. Consent was obtained from all the parents/guardians of the participating children and assent from the children was obtained prior to the study.

\section{RESULTS AND DISCUSSION}

\section{Demographic information}

Seven schools were included in the study and all of the children were in Grade R. The largest part of the sample was from the two schools from which the most parent consent forms were obtained (76.5\% of the sample; $n=52)$. Of the 68 children, $40(58.8 \%)$ were girls. The sample consisted of a heterogeneous group of 61 $(89.7 \%)$ black, five $(7.4 \%)$ coloured and two $(2.9 \%)$ white children. The median age was 5 years 8 months, ranging between 5 years 6 months and 5 years II months.

\section{Table I: The Beery VMI-6 subtests, overall scores and comparison of gender differences}

\begin{tabular}{|c|c|c|c|c|c|c|c|c|c|c|c|c|}
\hline \multirow{4}{*}{ Subtest } & \multicolumn{9}{|c|}{ Median (range) } & \multirow{2}{*}{\multicolumn{3}{|c|}{$\begin{array}{c}95 \% \mathbf{C l}^{*} \\
\text { (median difference for } \\
\text { gender) }\end{array}$}} \\
\hline & \multicolumn{3}{|c|}{ Total group $(n=68)$} & \multicolumn{3}{|c|}{ Girls $(n=40)$} & \multicolumn{3}{|c|}{ Boys $(n=28)$} & & & \\
\hline & \multicolumn{3}{|c|}{ Score } & \multicolumn{3}{|c|}{ Score } & \multicolumn{3}{|c|}{ Score } & \multicolumn{3}{|c|}{ Score } \\
\hline & Raw & Standard & Scaled & Raw & Standard & Scaled & Raw & Standard & Scaled & Raw & Standard & Scaled \\
\hline \multirow{2}{*}{$\begin{array}{l}\text { Motor } \\
\text { coordination } \\
(\mathrm{MC})\end{array}$} & 16 & 98 & 10 & 17 & 103.5 & 10.5 & 15 & 94 & 9 & I; 3* & $3 ; 12 *$ & $0 ; 2$ \\
\hline & $\begin{array}{c}(11- \\
24)\end{array}$ & $(76-133)$ & $(5-17)$ & $\begin{array}{l}(13- \\
24)\end{array}$ & $(86-133)$ & $(7-17)$ & $\begin{array}{c}(11- \\
22)\end{array}$ & $(76-123)$ & $(5-15)$ & & & \\
\hline \multirow{2}{*}{$\begin{array}{l}\text { Visual-motor } \\
\text { integration } \\
\text { (VMI) }\end{array}$} & 15 & 96 & 9 & 15 & 96 & 9 & 15 & 96.5 & 9 & $-1 ; 1$ & $-4 ; 4$ & $-I ; I$ \\
\hline & $\begin{array}{l}(9- \\
21)\end{array}$ & $(72-121)$ & $(4-14)$ & $\begin{array}{l}(9- \\
21)\end{array}$ & $(72-121)$ & $(4-14)$ & $\begin{array}{c}(10- \\
19)\end{array}$ & $(75-114)$ & $(5-13)$ & & & \\
\hline \multirow{2}{*}{$\begin{array}{l}\text { Visual } \\
\text { perception } \\
\text { (VP) }\end{array}$} & 15 & 92 & 8 & 15 & 92 & 8 & 15 & 92 & 8 & $-2 ; 1$ & $-10 ; 4$ & $-2 ; 1$ \\
\hline & $\begin{array}{l}(6- \\
26)\end{array}$ & $(45-136)$ & $(-I-17)$ & $\begin{array}{l}(6- \\
26)\end{array}$ & $(45-136)$ & $(-1-17)$ & $\begin{array}{c}(11- \\
22)\end{array}$ & $(75-120)$ & $(5-14)$ & & & \\
\hline \multirow{2}{*}{ Overall } & & 97.2 & & & 97.7 & & & 96 & & & $-3.3 ; 5$ & \\
\hline & & $(73-121)$ & & & $(73-121)$ & & & $\begin{array}{l}(79.7- \\
107.3)\end{array}$ & & & & \\
\hline \multicolumn{13}{|c|}{ *A significant difference is found when 0 does not fall within the confidence interval bracket. } \\
\hline Note: accorc & o th & merican & of the & ry & 6 test, s & score & torr & & & & & nor \\
\hline
\end{tabular}




\section{Beery VMI-6}

Table $I$ on page 20 indicates that for all the subtests of the Beery VMI-6, the children scored below the American standard score (SS) norm of 100 , but within the normative range of 85 tol $15^{6}$. Children performed the best in the motor coordination subtest (SS 98), followed by the visual-motor integration (SS 96) and the visual perception (SS 92) subtests.

These findings were similar to those reported by Doney et $\mathrm{al}^{54}$, who found that Aboriginal children in a remote region of Australia obtained slightly lower mean standard scores (motor coordination SS 95. I; visual perception SS 97.9; and visual-motor integration SS 89.6) than the American norms, but within the range of 85 to I I5. Conversely, Green et al. ${ }^{55}$ reported that a group of typical developing American children obtained higher mean visual-motor integration standard scores (105.17). The mean visual-motor integration scaled score of 9 measured in this study was similar to a previous South African study on the same test version, in which the children obtained a scaled score for visual-motor integration of $9.47^{41}$.

A statistically significant difference was found in the motor coordination subtest when comparing the genders, with girls performing better. This finding was comparable to a similar study $y^{20}$ where better performance was noticed among girls in the visual-motor integration sub-test, which had been attributed to the girls' tendency to be more cautious and slower in performing these visual-motor tasks ${ }^{20}$. $\mathrm{Parsa}^{56}$ also found that girls are prone to choose more figurative, soothing and fine motor activities, in comparison to boys who tend to participate in lively activities (also called gross motor activities). These gender differences imply that therapists should take this finding into account in clinical practice.
Based on these findings, the Beery VMI- 6 appears to be a suitable instrument for this sample of SA children, not displaying extreme performance differences in any of the subtests ${ }^{31}$. The implication for clinical practice is that the Beery VMI-6 may be used as a good initial measurement instrument ${ }^{31}$ as part of a comprehensive assessment.

\section{DTVP-3}

Table II below summarises the median scaled scores obtained by children for the eye-hand coordination subtest (SS 9), copying (SS I0), figure-ground (SS 8) and form constancy (SS 8.5), as compared to the American normative scaled score of 10 for all these subtests and a normative range of 7 to 13 .

The eye-hand coordination subtest in the DTVP-3 (SS 9) had a lower normative median scaled score compared to the American norm, but was consistent with Harris ${ }^{41}$ who obtained a scaled score of 8.7 in the DTVP-3 for children aged 6-9 years and Visser ${ }^{37}$, who established a mean scaled score of 9.2 in South African children aged 5 years and 6 years, using the DTVP-2. On the contrary, Smith ${ }^{36}$ and Visser et al. ${ }^{38}$ found that their South African sample scored higher on the eye-hand coordination subtest of the DTVP-2 than the American normative sample.

The results on the copying subtest (SS I0) were similar to those reported by Smith ${ }^{36}$, with a scaled score of 9.3 on the DTVP-2. However, Harris ${ }^{41}$ obtained a higher scaled score of I I. I for copying in the DTVP-3. The figure-ground (SS 8) and form constancy (SS 8.5) subtests' results of the DTVP-3 were lower in comparison to a previous study using the DTVP-2, for which scaled scores of 10.5 and II.8, respectively, were obtained ${ }^{36}$. This finding could be indicative of these specific subtests probably being more difficult to perform on the DTVP-3 compared to the previous version

Table II: The DTVP-3 subtests, composite scores and comparison of gender differences

\begin{tabular}{|c|c|c|c|c|c|c|c|c|}
\hline \multirow{3}{*}{ Subtests } & \multicolumn{6}{|c|}{ Median (range) } & \multirow{3}{*}{\multicolumn{2}{|c|}{$\begin{array}{l}95 \% \mathbf{C l} \\
\text { (median difference } \\
\text { for gender) }\end{array}$}} \\
\hline & \multicolumn{2}{|c|}{ Total group $(n=68)$} & \multicolumn{2}{|c|}{ Girls $(n=40)$} & \multicolumn{2}{|c|}{ Boys $(n=28)$} & & \\
\hline & Raw & Scaled & Raw & Scaled & Raw & Scaled & & \\
\hline \multirow{2}{*}{$\begin{array}{l}\text { Eye-hand } \\
\text { coordination(EH) }\end{array}$} & 145.5 & 9 & 145.5 & 9 & 145.5 & 9 & {$[-13 ; 9]$} & {$[-2 ; 1]$} \\
\hline & $(82-180)$ & $(3-14)$ & $(82-178)$ & $(3-14)$ & $(113-180)$ & $(6-14)$ & & \\
\hline \multirow[t]{2}{*}{ Copying (CO) } & 18 & 10 & 18.5 & 10 & 18 & 10 & {$[-1 ; 5]$} & {$[0 ; 3]$} \\
\hline & $(8-117)$ & $(5-19)$ & $(8-117)$ & $(5-19)$ & $(9-3 I)$ & $(5-18)$ & & \\
\hline \multirow{2}{*}{$\begin{array}{l}\text { Figure-ground } \\
\text { (FG) }\end{array}$} & 33 & 8 & 31.5 & 8 & 34 & 8 & {$[-4 ; 7]$} & {$[-1 ; 2]$} \\
\hline & $(6-54)$ & $(2-14)$ & $(17-54)$ & $(4-14)$ & $(6-49)$ & $(2-13)$ & & \\
\hline \multirow{2}{*}{$\begin{array}{l}\text { Visual closure } \\
\text { (VC) }\end{array}$} & 7 & 7.5 & 6.5 & 7 & 8 & 8.5 & {$[-2 ; 0]$} & {$[-2 ; 0]$} \\
\hline & $(2-14)$ & $(3-13)$ & $(2-14)$ & $(3-13)$ & $(3-14)$ & $(4-13)$ & & \\
\hline \multirow{2}{*}{$\begin{array}{l}\text { Form constancy } \\
\text { (FC) }\end{array}$} & 28 & 8.5 & 28.5 & 9 & 28 & 8 & {$[-2 ; 4]$} & {$[-1 ; 2]$} \\
\hline & $(17-40)$ & $(3-18)$ & $(21-40)$ & $(5-18)$ & $(17-36)$ & $(3-13)$ & & \\
\hline $\begin{array}{l}\text { DTVP-3 } \\
\text { composite } \\
\text { scores Comp: } \\
\text { composite) }\end{array}$ & Comp & $\begin{array}{l}\text { Sum of } \\
\text { scaled }\end{array}$ & Comp & $\begin{array}{l}\text { Sum of } \\
\text { scaled }\end{array}$ & Comp & $\begin{array}{l}\text { Sum of } \\
\text { scaled }\end{array}$ & Comp & Scaled \\
\hline \multirow{2}{*}{$\begin{array}{l}\text { Visual motor } \\
\text { integration (VMI) }\end{array}$} & 98.5 & 20 & 98.5 & 19.5 & 98.5 & 20 & {$[-3 ; 9]$} & {$[-2 ; 3]$} \\
\hline & $(76-127)$ & $(12-29)$ & $(82-127)$ & $(14-29)$ & $(76-118)$ & $(12-27)$ & & \\
\hline \multirow{2}{*}{$\begin{array}{l}\text { Motor-reduced } \\
\text { visual perception } \\
(\mathrm{MRP})\end{array}$} & 88 & 24 & 89 & 24.5 & 86 & 23 & {$[-4 ; 7]$} & {$[-2 ; 4]$} \\
\hline & $(67-116)$ & $(14-38)$ & $(67-116)$ & $(14-38)$ & $(67-108)$ & $(14-34)$ & & \\
\hline \multirow{2}{*}{$\begin{array}{l}\text { General visual } \\
\text { perception (GVP) }\end{array}$} & 93 & 44 & 95 & 46 & 91 & 43 & {$[-3 ; 6]$} & {$[-3 ; 6]$} \\
\hline & $(76-116)$ & $(3 \mid-62)$ & $(78-116)$ & $(32-62)$ & $(76-112)$ & $(31-59)$ & & \\
\hline \multicolumn{9}{|c|}{ Note: according to the American norm of the DTVP-3, scaled score - norm is 10 with a standard deviation of 3} \\
\hline \multicolumn{9}{|c|}{$\begin{array}{l}V M I=E H+C O \\
M R P=F G+V C+F C \\
G V P=E H+C O+F G+V C+F C\end{array}$} \\
\hline
\end{tabular}


of the test. Harris ${ }^{41}$, however, obtained higher scaled scores for figure-ground (10.2) and form constancy (I0.2) with the DTVP-3.

Compared to results from the other subtests, the children's performance in the visual closure subtest did not fall within the normative range and yielded the lowest score (SS 7.5). This observation was reported earlier in South African studies on the DTVP-2, and questioned the contextual appropriateness of specifically the visual closure subtests ${ }^{36-38}$. In all three of these studies visual closure scores were below the normative range for these South African samples. However, this subtest was not removed, the item linearity was changed and a scaled score of 9.7 was obtained by Harris ${ }^{41}$ on the DTVP-3 in SA. Further investigation is however recommended.

The children's median composite scaled scores for visual-motor integration (98.5), motor-reduced visual perception (88) and general visual perception (93) scored below the American norm of 100 but within the normative range of 85 to II 5 . When comparing genders, it was noted that for the subtests on eye-hand coordination, copying and figure-ground, both boys and girls obtained the same median scaled scores. There was a tendency, however, for boys to perform better than the girls on the visual closure subtest. Although girls performed better with regard to form constancy, motor-reduced visual perception and general visual perception, none of these gender differences were statistically significant.

\section{TVPS-3}

According to the data shown in Table III below, all the children obtained a median scaled score lower than the normative range (SS 8 to 10), and lower than the American norm of 10 on six of the seven subtests, namely visual discrimination SS 7), special relations (SS 6), form constancy (SS 6), sequential memory (SS 6), figureground (SS 7) and visual closure (SS 7). The children obtained a median scaled score within the normative range only in the visual memory subtest (SS 8).

It is evident from the results that between the tree tests, the children's performance on the TVPS- 3 were the lowest, and therefore can perhaps be considered as the most difficult of the three tests. The clinical implication of this finding is that therapists should be aware of the findings and should therefore not use the TVPS-3 as their only visual perceptual assessment instrument.

However, higher scaled scores were obtained on all the subtests in another South African study on the TVPS- $3^{41}$, with scaled scores ranging between 8.8 for both visual discrimination and form constancy and I 3 .I for spatial relations. Critical investigative questions need to be asked in order to understand possible reasons for the large differences observed in the specific population of South African children investigated in this study. The children's poor performance on this test cannot be ascribed to fatigue, since this was the first test administered.

Table III: The TVPS-3 subtests and composite scores, comparison of gender differences

\begin{tabular}{|c|c|c|c|c|c|c|c|c|}
\hline \multirow{3}{*}{ Subtests } & \multicolumn{6}{|c|}{ Median (range) } & \multirow{2}{*}{\multicolumn{2}{|c|}{$\begin{array}{c}\mathbf{9 5 \%} \mathbf{C l} \\
\text { (median difference for } \\
\text { gender) }\end{array}$}} \\
\hline & \multicolumn{2}{|c|}{ Total group $(n=68)$} & \multicolumn{2}{|c|}{ Girls $(n=40)$} & \multicolumn{2}{|c|}{ Boys $(n=28)$} & & \\
\hline & Raw & Scaled & Raw & Scaled & Raw & Scaled & Raw & Scaled \\
\hline \multirow[t]{2}{*}{ Visual discrimination (VD) } & 3 & 7 & 3 & 7 & 3 & 7 & {$[-1 ; 1]$} & {$[-I ; I]$} \\
\hline & $(1-8)$ & $(3-12)$ & $(1-8)$ & $(3-12)$ & $(1-8)$ & $(3-12)$ & & \\
\hline \multirow[t]{2}{*}{ Visual memory (VM) } & 5 & 8 & 5.5 & 10 & 5 & 8 & {$[-1 ; 2]$} & {$[-2 ; 3]$} \\
\hline & $(0-12)$ & $(0-25)$ & $(0-I I)$ & $(0-25)$ & $(0-12)$ & $(0-18)$ & & \\
\hline \multirow[t]{2}{*}{ Spatial relations $(S R)$} & 2 & 6 & 2 & 6.5 & 2 & 6 & {$[-1 ; 1]$} & {$[-1 ; 3]$} \\
\hline & $(0-12)$ & $(0-25)$ & $(0-1 \mathrm{I})$ & $(0-25)$ & $(0-12)$ & $(0-17)$ & & \\
\hline \multirow[t]{2}{*}{ Form constancy (FC) } & 3 & 6 & 3 & 6 & 3 & 6 & {$[0 ; 2]$} & {$[0 ; 3]$} \\
\hline & $(0-8)$ & $(0-25)$ & $(1-8)$ & $(I-25)$ & $(0-8)$ & $(0-14)$ & & \\
\hline \multirow[t]{2}{*}{ Sequential memory (SM) } & 2 & 6 & 2 & 6 & 1.5 & 5.5 & {$[0 ; 2]$} & {$[0 ; 5]$} \\
\hline & $(0-11)$ & $(0-17)$ & $(0-11)$ & $(0-17)$ & $(0-9)$ & $(0-14)$ & & \\
\hline \multirow[t]{2}{*}{ Figure-ground (FG) } & 3 & 7 & 3 & 7 & 3.5 & 8 & {$[-1 ; 0]$} & {$[-2 ; 0]$} \\
\hline & $(1-9)$ & $(3-16)$ & $(1-8)$ & $(3-14)$ & $(I-9)$ & $(3-16)$ & & \\
\hline \multirow[t]{2}{*}{ Visual closure (VC) } & 2 & 7 & 2 & 7 & 2 & 7 & {$[-1 ; 0]$} & {$[-2 ; 0]$} \\
\hline & $(0-7)$ & $(0-13)$ & $(0-6)$ & $(0-1 \mathrm{I})$ & $(0-7)$ & $(0-13)$ & & \\
\hline $\begin{array}{l}\text { Composite index scores } \\
\text { (SS: scaled score) }\end{array}$ & $\begin{array}{l}\text { Sum of } \\
\text { scaled }\end{array}$ & SS & $\begin{array}{l}\text { Sum of } \\
\text { scaled }\end{array}$ & SS & $\begin{array}{l}\text { Sum of } \\
\text { scaled }\end{array}$ & SS & $\begin{array}{l}\text { Sum of } \\
\text { scaled }\end{array}$ & SS \\
\hline \multirow[t]{2}{*}{ Overall index score I } & 49 & 85 & 49.5 & 85.5 & 45.5 & 84 & {$[-5 ; 8]$} & {$[-4 ; 5]$} \\
\hline & $(23-76)$ & $(67-I \mid 8)$ & $(26-73)$ & $(69-118)$ & $(23-76)$ & $(67-104)$ & & \\
\hline \multirow[t]{2}{*}{ Basic processes } & 28 & 84.5 & 29.5 & 86 & 27 & 83.5 & {$[-2 ; 6]$} & {$[-1 ; 9]$} \\
\hline & $(10-47)$ & $(62-109)$ & $(14-45)$ & $(67-106)$ & $(10-47)$ & $(62-109)$ & & \\
\hline \multirow[t]{2}{*}{ Sequencing } & 6 & 80 & 6 & 80 & 5.5 & 75 & {$[0 ; 5]$} & {$[0 ; 25]$} \\
\hline & $(0-17)$ & $(0-135)$ & $(0-17)$ & $(0-135)$ & $(0-14)$ & $(0-120)$ & & \\
\hline \multirow[t]{2}{*}{ Complex processes } & 14 & 85 & 14 & 85 & 16 & 86.5 & {$[-4 ;-1]$} & {$[-10 ; 1]$} \\
\hline & $(3-23)$ & $(58-108)$ & $(3-23)$ & $(58-108)$ & $(7-23)$ & $(61-103)$ & & \\
\hline \multicolumn{9}{|c|}{ Note: according to the American norm of the TVPS-3 the scaled score - norm is 10 with a standard deviation of 3} \\
\hline \multicolumn{9}{|c|}{$\begin{array}{l}\text { Basic processes }=\mathrm{VD}+\mathrm{VM}+\mathrm{SR}+\mathrm{FC} \\
\text { Sequencing }=\mathrm{SM} \\
\text { Complex processes }=\mathrm{FG}+\mathrm{VC}\end{array}$} \\
\hline
\end{tabular}


The spatial relations subtest requires the children to understand and comprehend the word "different". In the Curriculum and Assessment Policy Statements of South Africa ${ }^{57}$, it is expected of a Grade $\mathrm{R}$ learner to match objects that belong together and compare objects showing differences. Therefore, it is expected of the children to understand the concept of "different" 57 . However, the median enrolment age for the ELOTL schools in our study was 4 months 8 days. It does not necessarily mean that the children have a rich academic vocabulary in their language of learning (for most in the sample possibly their second language), in order for them to understand the term "different". This subtest provides two examples before the answers are scored, with the first example being a simple item and the second example being very complex. It could possibly be argued that this subtest shows a lack of linearity (meaning that levels of item difficulty are not gradually progressive).

The form constancy subtest included unfamiliar and complex shapes that the children are possibly not exposed to in class, which may make the subtest difficult for the sample population. The children's lower performance in the sequential memory subtest possibly could be attributed to not fully understanding the instructions, as well as the increasing number of items to remember within the same time limit, which might be an activity that is not practiced in class. Boys scored significantly higher than girls for the composite score of complex processes $(95 \% \mathrm{Cl}-4 ;-\mathrm{I})$.

Herbst and Huysamen ${ }^{34}$ reported a difference between the results of children from similar backgrounds but with different levels of exposure to appropriate pre-school experiences, and emphasise the importance of using culture-specific tests.

On a pre-school level, emphasis is often placed on the achievement and outcome of school-related activities such as letter and number formation, and mathematics. However, the importance of underlying visual perceptual skills development does not necessarily receive the same degree of emphasis. Furthermore, focus is put on activities such as "tracing" and "copying" and may be an explanation for children performing better overall on the motor tasks in this study, compared to the visual perceptual motor-free tasks. The visual perceptual skills development of children is not an automatic achievement (such as the milestone of sitting, for example), if the opportunity for learning has not been created in the home or educational setting, and the experience is not internalised. Investment in more organised learning by means of work sheets, visual perceptual activities and games is essential for the development of visual perceptual skills; hence the importance of class activities, the environment and also the educators acting as role models, to stimulate the development of an aspect such as visual perception ${ }^{58}$.

\section{Comparison of the subtests of the three tests}

In Table IV below, the results of subtests of the three tests that were comparable are summarised. The visual perceptual supplementary test of the Beery VMI- 6 is a single subtest and was therefore not compared to the multiple subtests comprising the composite visual perceptual component of the other two tests. This difference between the tests prevented the comparison of the children's performance on the Beery VMI-6 with the DTVP-3 and TVPS-3 with regard to visual perception.

A statistically significant difference was found when the form constancy subtests of the DTVP-3 (SS 8.5) and the TVPS-3 (SS 6) were compared. This finding could possibly be attributed to the fact that the DTVP-3 uses basic and familiar geometric shapes, such as circles and squares, whereas the TVPS- 3 uses more complicated shapes and unfamiliar figures and patterns.

Children also performed better in the figure-ground subtest of the DTVP-3 (SS 8) than that of the TVPS-3 (SS 7). Although this difference was not significantly different, the clinical significance needs to be considered since a single point-scaled score consists of several items. For the visual closure subtest, similar results were obtained with both the DTVP-3 (SS 7.5) and the TVPS-3 (SS 7).

A statistically significant difference was calculated between the motor coordination subtest of the Beery VMI- 6 and the eye-hand coordination subtest of the DTVP-3, where the children performed better in the Beery motor coordination subtest. Several factors were identified that might contribute to this finding. Firstly, the parallel lines between which the children had to draw were spaced further apart in the Beery VMI-6 subtest than those of the DTVP-3. Secondly, for the DTVP-3 subtest, the children scored zero out of a total of 4 when they picked up their pencils. Since it was found that the children often picked up their pencil, this influenced their scores negatively, whereas this rule does not apply to the Beery VMI-6.

When the visual motor integration subtest of the Beery VMI-6 was compared to the copying subtest of the DTVP-3, no statistically significant difference was found. However, the children performed better in the DTVP-3 subtest, which also puts focus on an important implication for clinical practice. This finding could be due to DTVP-3 copying subtest allowing the child to score between 0 and 2 , with more lenient scoring criteria than the Beery VMI-6, where the child can only score 0 or I, with each item having very specific marking criteria.

\section{Comparison of the composite scores}

The total of all of the subtests' scores represent the overall scores. When the median overall score of the Beery VMI- 6 was compared to that of the DTVP-3, the children performed significantly better overall on the Beery VMI-6 $(95 \% \mathrm{Cl}$ I; 4.7). These findings could be

Table IV: Comparison of results for comparable subtests scaled scores of the three tests for visual perception

\begin{tabular}{|c|c|c|c|c|c|c|}
\hline Beery VMI-6 & $\begin{array}{l}\text { Median } \\
\text { (range) }\end{array}$ & DTVP-3 & Median (range) & TVPS-3 & $\begin{array}{l}\text { Median } \\
\text { (range) }\end{array}$ & $\begin{array}{c}95 \% \mathrm{Cl} \\
\text { for median } \\
\text { differences }\end{array}$ \\
\hline \multicolumn{7}{|l|}{ Non-motor components } \\
\hline \multirow[t]{3}{*}{ Visual closure (VC) } & & $\begin{array}{l}\text { Form constancy } \\
(\mathrm{FC})\end{array}$ & $\begin{array}{c}8.5 \\
(3-18)\end{array}$ & $\begin{array}{c}\text { Form } \\
\text { constancy }(\mathrm{FC})\end{array}$ & $\begin{array}{c}6 \\
(0-25)\end{array}$ & {$[1 ; 4] *$} \\
\hline & & Figure-ground (FG) & $\begin{array}{c}8 \\
(2-14)\end{array}$ & $\begin{array}{l}\text { Figure-ground } \\
\text { (FG) }\end{array}$ & $\begin{array}{c}7 \\
(3-16)\end{array}$ & {$[-2 ; 0]$} \\
\hline & & Visual closure (VC) & $\begin{array}{c}7.5 \\
(3-13)\end{array}$ & $\begin{array}{l}\text { Visual closure } \\
\text { (VC) }\end{array}$ & $\begin{array}{c}7 \\
(0-13)\end{array}$ & {$[0 ; 3]$} \\
\hline \multicolumn{7}{|l|}{ Motor components } \\
\hline \multirow[t]{2}{*}{ Motor coordination (MC) } & $\begin{array}{c}10 \\
(5-17)\end{array}$ & $\begin{array}{c}\text { Eye-hand } \\
\text { coordination }(\mathrm{EH})\end{array}$ & $\begin{array}{c}9 \\
(3-14)\end{array}$ & & & {$[0 ; 2]$} \\
\hline & 9 & Copying & 10 & & & {$[-2 ; 0]$} \\
\hline Visual-motor integration (VMI) & $(4-14)$ & & $(5-19)$ & & & \\
\hline
\end{tabular}


due to the Beery VMI-6 having three subtests compared to five in the DTVP-3. Furthermore, two of the three Beery VMI-6 subtests require a motor output, whereas a motor output is required for only two of the five DTVP-3 subtests.

The median overall score of the Beery VMI- 6 showed that the children performed significantly better in this test, compared to the TVPS-3 $(95 \% \mathrm{Cl} 10.3 ; 15.3)$. A possible explanation for this finding could be that the children found the non-motor twodimensional based shapes and concepts used in the TVPS-3 more challenging.

When comparing the DTVP-3 to the TVPS-3, it was found that the children performed significantly better overall on the DTVP-3 $(95 \% \mathrm{Cl} 7 ; \mathrm{II})$. This finding could possibly be due to insufficient exposure to complex two-dimensional visual perceptual activities in the current curriculum and/or limitations in the teachers' repertoire. It could also be attributed to the TVPS-3 having more subtests, or the absence of motor components in the TVPS-3 may have a substantial influence on the child's performance.

Atkinson and Braddick (cited by Tsaia $^{26}$ ) reported that visual perception is a developing process that is very well developed by the age of 12 years. In our study, the sample population was at an age where they were still developing these skills.

\section{LIMITATIONS, IMPLICATIONS FOR PRACTICE AND RECOMMENDATIONS}

The limitations associated with this study were, firstly, that convenience sampling was used for including the children into the study and consequently, they were not sampled randomly; hence, a larger proportion of children were sourced from two of the participating schools. Secondly, due to the poor response rate and limited data collection time, an amendment was made to the original protocol and children who attended an ELOLT school for the median of 4 months 8 days were included, opposed to the initial inclusion criterion of at least one year. Thirdly, differences in the school settings and educational backgrounds among children were not taken into account. Finally, no differences other than language and ethnicity have been taken into account in this small sample.

Despite these limitations, the implication for practice is that the results can only be generalised to similar South African populations. Recommendations with regard to clinical practice include the following:

* Since the latest editions of the Beery VMI-6, DTVP-3 and TVPS-3 visual perceptual tests have been developed based on research and specific reviews and feedback from the users recognising these new versions as being an improvement, clinical occupational therapy practices should consider purchasing these tests.

* Occupational therapists should apply, interpret and convey the results of these tests with caution.

* Although children's subtest performances are valuable in the evaluation and interpretation of results, important decisions with regard to intervention should rest primarily on the composite indexes of these tests.

* Undergraduate students should be trained in the use of a variety of instruments (preferably on the latest editions), the limitations of the instruments and on the validity and reliability of a test so that they can choose the best one.

* These tests' prescribed instructions should be translated into the South African official languages in order to avoid conceptual misunderstanding, which might affect test results.

* The TVPS-3 should not be used as the only visual perceptual test in the assessment process to guide intervention planning, but should be used in combination with the Beery VMI-6 and/ or DTVP-3.

* These tests aim to assess children's skills and do not take into consideration cogent factors, such as the child's living and educational environment, personal factors, and activityparticipation features ${ }^{14}$. Therefore, these tests need to be used in conjunction with other activity-participation assessment instruments.

This study provides preliminary data as a reference for future studies. Based on the results and conclusions drawn from this research, the authors make the following recommendations with regards to future research on the Beery VMI-6, DTVP-3 and TVPS-3:

* An investigation into the validity and reliability of these tests on larger samples representing different cultural and language groups and from different educational settings in South Africa; * the development of contextual-specific instruments; and

* the standardisation of existing international instruments in the South African context.

\section{CONCLUSION}

This study describes the visual perception and visual-motor performance of a sample of South African five-year-old, English-speaking children by means of the Beery VMI-6, DTVP-3 and TVPS-3 visual perceptual tests. The findings provide occupational therapists using these tests with evidence of how the norms of the South African study sample compare to those of the American normative sample. Although these tests are considered as standardised measuring instruments, commonly used internationally, it is evident that certain aspects of these tests are unsuitable in its current state. While no context-specific visual perceptual measuring instruments instrument is currently available in South Africa, occupational therapists should be mindful of the manner in which they use these tests, interpret results and make recommendations for practice.

\section{ACKNOWLEDGEMENTS}

The participants, their parents and school staff; Dr Daleen Struwig, medical writer/editor, Faculty of Health Sciences, University of the Free State, for technical and editorial preparation of the manuscript.

\section{REFERENCES}

I. Martin NA. Test of visual perceptual skills (TVPS-3). $3^{\text {rd }}$ ed. Novato, CA: Academic Therapy Publications; 2006.

2. Brown T and Rodger S. The profile of Canadian paediatric occupational therapy practice. Occupational Therapy in Health Care. 2007; 21 : 39-69.

3. Brown T, Rodger S, Brown A and Roever C. A comparison of Canadian and Australian paediatric occupational therapists. Occupational Therapy International. 2005; 12: 137-6I.

4. Diamantis AD. Use of standardised tests in paediatrics: The practice of private occupational therapists working in the United Kingdom. British Journal of Occupational Therapy. 2006; 69: 28I-7.

5. Van der Merwe J, Smit N and Vlok B. A survey to investigate how South African occupational therapists in private practice are assessing and treating poor handwriting in foundation phase learners: Part I demographics and assessment practices. South African Journal of Occupational Therapy. 20II; 4I: 3-II.

6. Beery KE and Beery NA. Administration, scoring and teaching manual for the Beery-VMI. $6^{\text {th }}$ ed. San Antonio, TX: Pearson; 2010.

7. Hammil D, Pearson $\mathrm{N}$ and Vores J. Developmental test of visual perception examiner's manual. $3^{\text {rd }}$ ed. Austin, TX: Pro-Ed; 2014.

8. Sortor JM and Kulp MT. Are the results of the Beery-Buktenica Developmental Test of Visual-Motor Integration and its subtests related to achievement test scores? Optometry and Vision Science. 2003; 80: 758-62.

9. Brown T, Elliot S, Bourne R, Sutton E, Wigg S, Morgan D, Glass S and Lalor A. The discriminative validity of three visual perceptual tests. New Zealand Journal of Occupational Therapy. 201 I; 58:14-22.

10. Brown T and Rodger S. An evaluation of the validity of the Test of Visual Perceptual Skills - Revised (TVPS-R) using the Rasch measurement model. British Journal of Occupational Therapy. 2009; 72: 65-78.

II. Brown T and Hockey SC. The validity and reliability of developmental test of visual perception- $2^{\text {nd }}$ edition (DTVP-2). Physical and Occupational Therapy in Paediatrics. 2013; 33: 426-39.

12. Brown T. Construct validity of the three motor-reduced subscales of the Developmental Test of Visual Perception - Adolescent and 
Adult (DTVP-A): a Rash analysis model evaluation. British Journal of Occupational Therapy. 20II; 74: 66-77.

13. Brown T, Mullins E and Stagnitti K. The reliability of performance of healthy adults on three visual perception tests. British Journal of Occupational Therapy. 2008; 7I: 438-47.

14. Brown T. Validity and reliability of the Developmental Test of Visual Perception - Third Edition. Occupational Therapy in Health Care. 2016; 30: 272-87.

15. Brown T and Murdolo Y. The Developmental Test of Visual Perception-Third Edition (DTVP-3): A review, critique, and practice implications. Journal of Occupational Therapy, Schools and Early Intervention. 20I5; 8: 336-54.

16. Brown T, Mullins E and Stagnitti K. The concurrent validity of three visual perception tests used with adults. Occupational Therapy in Health Care. 2009; 23: 99-II8.

17. Brown T, Bourne R, Sutton E, Wigg S, Burgess D, Glass S, Elliott S and Lalor A. The reliability of three visual perception tests used to assess adults. Perceptual Motor Skills. 20 10; III: 45-59.

18. Lim CY, Tan PC, Koh C, Koh E, Guo H, Yusoff ND, See CQ and Tan T. Beery-Buktenica Developmental Test of Visual-Motor Integration (Beery-VMI): lessons from exploration of cultural variations in visual-motor integration performance of pre-schoolers. Child: Care, Health and Development. 20I4; 4I: 213-2I.

19. Lai MY and Leung FKS. Visual perceptual abilities of Chinesespeaking and English-speaking children. Perceptual and Motor Skills. 2012; 114: 433-45.

20. Cheung P, Poon M, Leung L and Wong R. The Developmental Test of Visual Perception-2 normative study on the visual-perceptual function for children in Hong Kong. Physical and Occupational Therapy in Paediatrics. 2006; 25: 29-43.

21. Ho W, Tang MM, Fu C, Leung K, Pang PC and Cheong AM. Relationship between vision and visual perception in Hong Kong preschoolers. Optometry and Vision Science. 2015; 92: 623-31

22. Josman N, Abdallah TM and Engel-Yeger B. A comparison of visual-perceptual and visual-motor skills between Palestinian and Israeli children. American Journal of Occupational Therapy. 2006; 60: 215-25.

23. Chiu E, Wu W, Chou C, Yu M and Hung J. Test-retest reliability and minimal detectable change of the Test of Visual Perceptual SkillsThird Edition in patients with stroke. Archives of Physical Medicine and Rehabilitation. 2016; 97: 1917-23.

24. Guntayuong C, Chinchai S, Pongsaksri M and Vittayakorn S. Determination of normative values of the Developmental Test of Visual Perception (DTVP-2) in Thai children. International Journal of Medicine and Pharmaceutical Sciences. 20I3; 3: I I3-26.

25. Auld M, Boyd R, Moseley L and Johnston L. Seeing the gaps: a systematic review of visual perception tools for children with hemiplegia. Disability and Rehabilitation. 20I I; 33: 1854-65.

26. Tsai LT, Lin KC, Liao HF and Hsieh CL. Reliability of two visualperceptual tests for children with cerebral palsy. American Journal of Occupational Therapy. 2009; 63: 473-80.

27. McDonald CA, Volker MA, Lopata C, Toomey JA, Thomeer ML, Lee GK, Lipinski AM, Dua EH, Schiavo AM, Bain F and Nelson AT. VMI-VI and BG-II KOPPITZ-2 for youth with HFASDs and typical youth. Journal of Psychoeducational Assessment. 20I4; 32: 379-89.

28. Bainbridge J. Visual perceptual processing and occupational performance in South African adults with traumatic brain injury - a validity study of the Test of Visual Perceptual skill-3. Master's dissertation. Johannesburg: University of the Witwatersrand, Johannesburg; 2015.

29. Idoni J, Taub MB and Harris PA. A comparison of two tests of visual-motor integration. Optometry and Visual Performance. 2014; 2: 170-4.

30. Brown T, Chinner A and Stagnitti K. Convergent validity of tow visual motor integration tests. British Journal of Occupational Therapy. 20II; 74 (6): 295-303.

31. McCrimmon AW, Altomoare AA and Matchullis TL. Test review: the Beery Developmental Test of Visual-Motor Integration $6^{\text {th }}$ edition. Journal of Psychoeducational Assessment. 2012: 30: 588-92.

32. Dunn M. The validity of the Developmental Test of Visual-Motor Integration in a selected pre-school sample in the South African context. Unpublished Master's dissertation. Stellenbosch: University of Stellenbosch; 2001.

33. Rens Z. The standardization of the Beery-Buktenica developmental test of visual-motor integration with supplemental developmental tests of visual perception and motor coordination ( $4^{\text {th }}$ edition, revised 1997) on an Eastern Cape population aged 7 years 0 months to 7 years 3 months. Master's degree disertation. Johannesburg: University of the Witwatersrand; 2009.

34. Herbst I and Huysamen G. The construction and validation of developmental scales for environmentally disadvantaged preschool children. South African Journal of Psychology. 2000; 30: 19-24.

35. Lotz L. Assessment of visual-motor integration functioning in a selected South African middle childhood sample. Master's dissertation. Stellenbosch: University of Stellenbosch; 2003.

36. Smith M. The suitability of the Developmental Test of Visual Perception $-2^{\text {nd }}$ Edition (DTVP-2) for 5-year old English-speaking children in urban South Africa. Master's dissertation. Bloemfontein: University of the Free State; 2015.

37. Visser MM. The association of an omitted crawling milestone on pencil grasp and control in a 5 \& 6 year old population. Master's research report. Johannesburg: University of Witwatersrand; 2004.

38. Visser $M$, Cronjé $M, \operatorname{Kemp} B$, Scholtz $M$, van Rooyen $W$ and Nel $M$. The DTVP-2 visual closure subtest: a closer look. South African Journal of Occupational Therapy. 20I2; 42: 2I-5.

39. Vorster M. Die gebruik van die Beery-ontwikkelingstoets en die natekentoets as meetinstrumente van visueel-motoriese integrasie by ' $n$ groep voorskoolse kinders. Master's dissertation. Stellenbosch: University of Stellenbosch; 1994.

40. Richmond J and Holland K. Correlating the Developmental Test of Visual Perception-2 (DTVP-2) and the Test of Visual Perceptual Skills-Revised (TVPS-R) as assessment tools for learners with difficulties. South African Journal of Occupational Therapy. 20 I I; 4I: 33-7.

4I. Harris $M$. The validity of standardised visual perceptual tests in identifying specific learning disabilities in children from Gauteng Province, South Africa. Presentation: $35^{\text {th }}$ Occupational Therapy Association (OTASA) Congress, WITS University; I4-16 July 2016.

42. American Occupational Therapy Association. Framework: Domain and Process, $3^{\text {rd }}$ ed. American Journal of Occupational Therapy. 20I4; 68(SuppII): SI-S48.

43. Richardson PK. Use of standardized tests in pediatric practice. In: Case-Smith J and O'Brien JC (eds.). Occupational Therapy for Children. $6^{\text {th }}$ ed. Maryland Heights, MO: Mosby, 2010: 216-39.

44. Herbst I. Early childhood development criteria manual. Bloemfontein: University of the Free State; 2006.

45. Schneider E, Parush S, Katz N and Miller L. Performance of Israeli versus U.S. preschool children on the Miller Assessment for Preschoolers. American Journal of Occupational Therapy. 1995; 49: 19-23.

46. Urbina S. Essentials of psychological testing. Hoboken, NJ: John Wiley \& Sons, Inc.; 2004.

47. Clarke, K. Construct validity of the Developmental Test of VisualPerception Third Edition (DTVP-3) in Western Australian primary school children. Bachelor of Science. Perth: Edith Cowan University; 2015. Retrieved from http://ro.ecu.edu.au/theses_hons/I474.

48. Republic of South Africa Department of Education. South African Schools Act, 1996 (Act No. 84 of 1996). Government Notice No. 1293 of 1998. Government Gazette, 1998; 400(I9377): I-5. <www.gov.za/sites/www.gov.za/files/Act84of 1996.pdf> (16 February 2017).

49. Gardner M. Test of Visual-Perceptual Skills (Non-Motor). San Francisco, CA: Psychological and Educational Publications; 1982.

50. Gardner M. Test of Visual Perceptual Skills (Non-Motor) - Revised. San Francisco, CA: Psychological and Educational Publications; 1996.

5I. Martin NA. Test of visual perceptual skills (TVPS-4). $4^{\text {rd }}$ ed. Novato, CA: Academic Therapy Publications; 2017.

52. Department of Basic Education. Government Gazette no 34346 of 6/6/20II, General Notice 487 of 201 I. SC006: Dictionary of Education Concepts and Terms; 2010.

53. Schlodder MI. The Beery Visual-Motor Integration test: a crossethnic comparison of normal preschool children. Master's degree disertation. Port Elizabeth: University of Port Elizabeth; 1986.

54. Doney R, Lucas BR, Watkins RE, Tsang TW, Sauer K, Howat P, Latimer J, Fitzpatrick JP, Oscar J, Carter M and Elliott EJ. Visual-motor integration, visual perception, and fine motor coordination in a population of children with high levels of fetal alcohol spectrum disorder. Research in Developmental Disabilities. 2016; 55: 346-57. 
55. Green RR, Bigler ED, Froehlich A, Prigge MB, Travers BG, Cariello AN, Anderson JS, Zielinski BA, Alexander A, Lange $N$ and Lainhart JE. Beery VMI performance in autism spectrum disorder. Child Neuropsychology. 2016; 22: 795-817.

56. Parsa M. Adolescence psychology. $3^{\text {rd }}$ ed. Tehran: Besat Press; 1988.

57. Curriculum and Policy Assessment Statement. National Curriculum Statement: Foundation Phase; 20II; 31.

58. Vygotsky LS. Mind in society: the development of higher psychological processes. Cambridge, MA: Harvard University Press; 1979.

\section{Corresponding Author}

\section{Marieta Visser}

Telephone: +2751 401 2831

Email address: vissermm@ufs.ac.za 